\title{
A Biliobronchial Fistula in a Patient With Hepatocellcular Carcinoma Treated With Chemoembolization Diagnosed by Hepatobiliary Iminodiacetic Acid Scan and Managed by Endoscopic Retrograde Cholangiopancreatography
}

\author{
Yousef Nassar, ${ }^{\mathrm{a}, \mathrm{b}}$, Sven Hida ${ }^{\mathrm{a}}$, Seth Richter ${ }^{\mathrm{a}}$
}

\begin{abstract}
A biliobronchial fistula is an abnormal communication between the biliary tract and the bronchial tree. It may be a rare complication after treatment of hepatocellular carcinoma with transcatheter arterial chemoembolization (TACE). We present a case of a 71-year-old man that developed a biliobronchial fistula as a complication of hepatocellular carcinoma treated with TACE. It was successfully diagnosed by hepatobiliary iminodiacetic acid (HIDA) scan and treated with endoscopic retrograde pancreatography.
\end{abstract}

Keywords: Biliobronchial fistula; Transcatheter arterial chemoembolization; Bilioptysis; ERCP; Hepatocellular carcinoma

\section{Introduction}

A biliobronchial fistula (BBF) is an abnormal communication between the biliary tract and the bronchial tree. This condition can be congenital or acquired. Acquired BBFs have many possible causes and may be the result of an obstruction of the biliary tract, a hepatic abscess, trauma, local infection such as hydatid cyst or amebic disease of the liver. It may be a rare complication of hepatic malignancies both metastatic and primary or as a complication of hepatocellular carcinoma treatments such as radiofrequency ablation (RFA) and transcatheter arterial chemoembolization (TACE) [1, 2]. Signs and symptoms of a BBF are variable and most patients typically present with bilioptysis or cough productive of bilious sputum and some may have a fever. Diagnosis may be established by advanced imaging studies such as hepatobiliary iminodiacetic acid (HIDA) scan or magnetic resonance cholangiopancrea-

Manuscript submitted September 1, 2017, accepted September 20, 2017

aAlbany Medical Center, Albany, NY, USA

${ }^{b}$ Corresponding Author: Yousef Nassar, Albany Medical Center, 43 New Scotland Avenue, MC 17, Albany, NY 12208, USA. Email: nassary@mail.amc.edu

doi: https://doi.org/10.14740/gr904w tography (MRCP). Management of BBFs can be extremely difficult and are often associated with high morbidity and mortality rates [3]. Suitable and prompt treatment is crucial; however, there are no consensus-based standard treatments for BBFs with the optimal choice depending on the underlying condition and the biliary tract $[4,5]$. Multiple treatment modalities have been used in cases of BBFs and reported in literature. Common treatment modalities that have been used in previous cases include endoscopic retrograde biliary drainage as well as surgery to treat the underlying etiology and manage the BBF. Percutaneous placement of an external biliary drain has been used in previously reported cases with success [1].

\section{Case Report}

A 71-year-old man presented to our hospital complaining of a cough productive of yellow colored sputum for 3 months prior to presentation. The cough was associated with weight loss as well as generalized fatigue but the patient denied having any fevers. His past medical history is significant for hepatocellular carcinoma, treated with TACE with doxorubicin 12 months prior to presentation. The patient was hospitalized at another hospital 3 months earlier when his cough started and was treated with antibiotics for suspected pneumonia and was discharged. His cough did not improve. Physical examination revealed dullness to percussion over the right lower lung field associated with rhonchi heard in the same field. Otherwise, examination was unremarkable. His initial presenting labs were normal. A right lower lobe consolidation was seen on chest X-ray. A sputum test for bilirubin revealed a level of 6.7 $\mathrm{mg} / \mathrm{dL}$.

An HIDA scan was performed which revealed a biliary leak into the right lower lung and trachea (Fig. 1). The biliary leak was found to be secondary to a BBF. During hospitalization, an endoscopic retrograde cholangiopancreatography (ERCP) was performed with sphincterotomy and a 7 Fr by 5 $\mathrm{cm}$ temporary stent was placed in the common bile duct to manage the BBF (Fig. 2). The patient's cough and biliary sputum improved significantly after the stent was placed and he currently no longer has a cough or bilious sputum. 


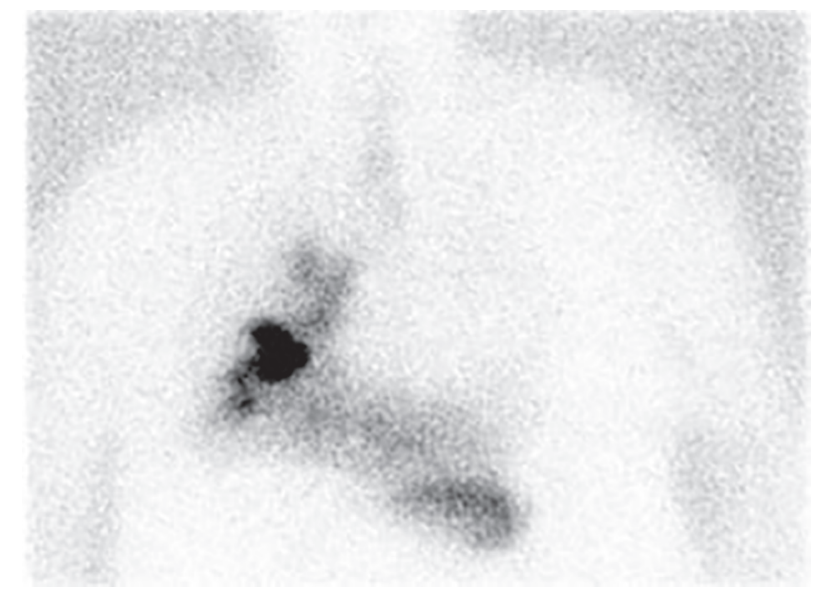

Figure 1. HIDA scan demonstrating biliary leak extending into the right lung and trachea.

\section{Discussion}

A BBF is an abnormal communication between the biliary tract and the bronchial tree. It may create a diagnostic dilemma in the presenting symptoms of patients as they may be similar to symptoms of a respiratory infection. Patients typically present with a cough which is usually productive of yellow colored sputum and some may have a fever. Initial assessment may include a chest X-ray that may reveal a consolidation or pleural effusion further contributing to misdiagnosing patients with a respiratory infection such as pneumonia. As was used in this case, an HIDA scan can be used in the diagnosis of a BBF by displaying the biliary leak in the lung. An alternative imaging modality that may establish the diagnosis is MRCP. This condition should be considered in the differential diagnosis of patients with persistent cough particularly those with a known history of conditions that pre-dispose patients to develop BBFs such as those with known hepatic malignancy, primary or metastatic [1]. A rapid and cost effective test as was used in this case to diagnose a BBF can be to test the sputum for bilirubin. BBFs have been reported as a complication of trauma [6], hepatocellular carcinoma that was previously treated with radioablation [7] and partial hepatectomy [4]. A $\mathrm{BBF}$ has been reported as a complication of hepatic hydatid cyst [3] as well as pyogenic liver abscess. In previously reported cases, the approach to managing BBF varied greatly. In a case reported by Gandhi et al of a BBF caused by traumatic injury, a surgical approach was used in which resection of the BBF was performed [8]. In another case reported by Hai et al, a BBF occurred as a result of partial hepatectomy used in the management of hepatocellular carcinoma [4]. In this case, surgical revision with resection of the BBF was required in order to manage it. Medical management using octreotide has been reported in a case by Ong et al, in which octreotide was used to decrease bilious cough in a patient and accelerate fistula closure but its use is limited and may be ineffective in cases involving underlying infection, malignancy or obstruction as BBFs remain patent due to these underlying causes and a definitive management approach is recommended [9].

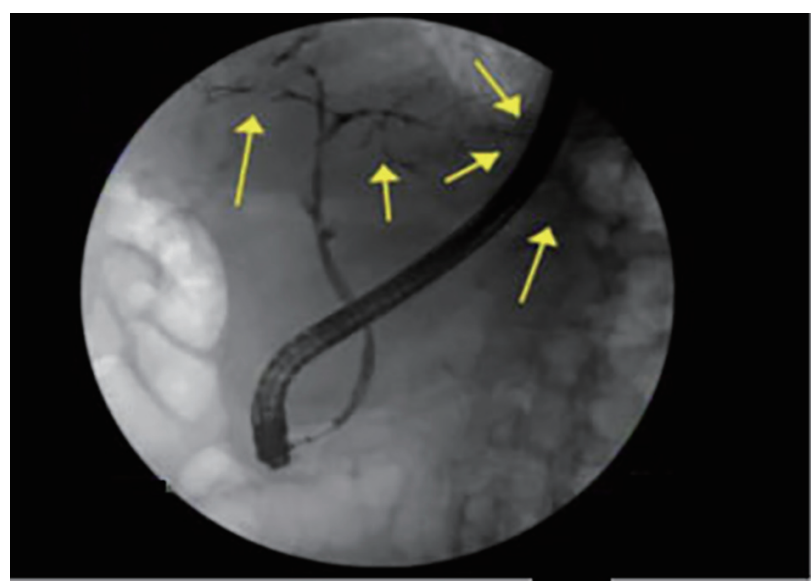

Figure 2. Left intrahepatic branches of the biliary tree-interrupted intrahepatics in both, left and right distribution with left-sided extravasation as demonstrated by yellow arrows.

In this case, a combination of ERCP with sphincterotomy and placement of a temporary common bile duct stent was used to manage a BBF that developed as a late complication of TACE used to manage hepatocellular carcinoma. This successfully managed the patient's symptoms, leading to complete resolution of symptoms and may allow the fistula to close by reducing biliary flow across the fistula. This can be an alternative to surgery though depending on the underlying cause, surgery may be a more appropriate treatment option and may allow surgical resection of an underlying malignancy.

\section{References}

1. Liao GQ, Wang H, Zhu GY, Zhu KB, Lv FX, Tai S. Management of acquired bronchobiliary fistula: A systematic literature review of 68 cases published in 30 years. World J Gastroenterol. 2011;17(33):3842-3849.

2. Yoon DH, Shim JH, Lee WJ, Kim PN, Shin JH, Kim KM. Percutaneous management of a bronchobiliary fistula after radiofrequency ablation in a patient with hepatocellular carcinoma. Korean J Radiol. 2009;10(4):411-415.

3. Senturk H, Mert A, Ersavasti G, Tabak F, Akdogan M, Ulualp K. Bronchobiliary fistula due to alveolar hydatid disease: report of three cases. Am J Gastroenterol. 1998;93(11):2248-2253.

4. Hai S, Iimuro Y, Hirano T, Suzumura K, Yada A, Fujimoto J. Bronchobiliary fistula caused after hepatectomy for hepatocellular carcinoma: a case report. Surg Case Rep. 2016;2(1):147.

5. Lee JH, Kim MS, Lee JG, Kim DS, Yang HJ, Cho DH, Kang KW. A case of bronchobiliary fistula as a complication of radiofrequency ablation. Tuberc Respir Dis. 2012;72(2):228-231.

6. Stowers J, Hill J, Straus J. Traumatic bronchobiliary fistula. Am Surg. 2014;80(6):E182-184.

7. Tran T, Hampel H, Qureshi WA, Shaib Y. Successful endoscopic management of bronchobiliary fistula due to radiofrequency ablation. Dig Dis Sci. 2007;52(11):3178- 
3180 .

8. Gandhi N, Kent T, Kaban JM, Stone M, Teperman S, Simon R. Bronchobiliary fistula after penetrating thoracoabdominal trauma: case report and literature review. J
Trauma. 2009;67(5):E143-145.

9. Ong M, Moozar K, Cohen LB. Octreotide in bronchobiliary fistula management. Ann Thorac Surg. 2004;78(4):1512-1513; author reply 1513. 